\title{
THE STRESS-ENERGY TENSOR FOR BIHARMONIC MAPS
}

\author{
E. LOUBEAU, S. MONTALDO, AND C. ONICIUC
}

\begin{abstract}
Using Hilbert's criterion, we consider the stress-energy tensor associated to the bienergy functional. We show that it derives from a variational problem on metrics and exhibit the peculiarity of dimension four. First, we use this tensor to construct new examples of biharmonic maps, then classify maps with vanishing or parallel stress-energy tensor and Riemannian immersions whose stress-energy tensor is proportional to the metric.
\end{abstract}

\section{INTRODUCTION}

As described by Hilbert in [16, the stress-energy tensor associated to a variational problem is a symmetric 2-covariant tensor $S$ conservative at critical points, i.e. $\operatorname{div} S=0$.

In the context of harmonic maps, i.e. critical points of the energy $E(\phi)=$ $\frac{1}{2} \int_{M}|d \phi|^{2} v_{g}$, the stress-energy tensor was studied in details by Baird and Eells in [1]. Indeed, the Euler-Lagrange equation associated to the energy is the vanishing of the tension field $\tau(\phi)=$ trace $\nabla d \phi$, and the tensor

$$
S=\frac{1}{2}|d \phi|^{2} g-\phi^{*} h
$$

satisfies $\operatorname{div} S=-\langle\tau(\phi), d \phi\rangle$.

As shown by Sanini in 24], $S$ vanishes precisely at critical points of the energy for variations of the domain metric, rather than variations of the map.

In this paper we consider a natural generalization of harmonic maps, suggested by Eells and Sampson [14]: the bienergy of $\phi:(M, g) \rightarrow(N, h)$ is

$$
E_{2}(\phi)=\frac{1}{2} \int_{M}|\tau(\phi)|^{2} v_{g}
$$

and a map is biharmonic if it is a critical point of $E_{2}$, equivalently, if it satisfies the associated Euler-Lagrange equation

$$
\tau_{2}(\phi)=-\Delta \tau(\phi)-\operatorname{trace} R^{N}(d \phi, \tau(\phi)) d \phi=0 .
$$

2000 Mathematics Subject Classification. 58E20.

Key words and phrases. Harmonic maps, biharmonic maps, stress-energy tensor.

The third author was supported by a CNR-NATO (Italy) fellowship. 
In [18, Jiang constructed an ad-hoc (0,2)-tensor

$$
\begin{aligned}
S_{2}(X, Y)= & \frac{1}{2}|\tau(\phi)|^{2}\langle X, Y\rangle+\langle d \phi, \nabla \tau(\phi)\rangle\langle X, Y\rangle \\
& -\left\langle d \phi(X), \nabla_{Y} \tau(\phi)\right\rangle-\left\langle d \phi(Y), \nabla_{X} \tau(\phi)\right\rangle,
\end{aligned}
$$

such that $\operatorname{div} S_{2}=-\left\langle\tau_{2}(\phi), d \phi\right\rangle$, thus conforming to the principle of a stressenergy tensor for the bienergy. In analogy with harmonic maps, one would expect that such a tensor could be depicted by metric variations. Theorem 2.3 shows that $S_{2}$ does indeed possess this property.

Motivated by this characterization, we study $S_{2}=0$ in details, and describe, in Section [3] situations for which this implies harmonicity of the map. On compact domains $(m \neq 4)$, this was already proved by Jiang. This specialness of dimension four is the subject of Theorem 3.12 and Proposition 3.13 where Riemannian and conformal immersions with $S_{2}=0$ are classified.

A salient feature of this tensor is its usefulness in finding new biharmonic maps. In fact, the search for maps with vanishing bitension field is replaced with one for divergence-free stress-energy tensor, as implemented in Proposition 2.6] and 2.12]

Since $S_{2}=0$ is a particularly strong condition, we study, in Section 4. maps with parallel stress-energy tensor. This clearly is stronger than being simply divergence free. In Theorem 4.4 we classify hypersurfaces with parallel stress-energy tensor and, in Proposition 4.5 characterize pseudo-umbilical Riemannian immersions with parallel stress-energy tensor.

Finally, we point out that, if $M$ is compact, the bienergy is homogeneous of degree zero with respect to the metric $g$ if and only if $m=4$. Therefore, if $m \neq 4$, changing homothetically the domain metric, we can render the bienergy arbitrarily large or small. This leads to considering volumepreserving variations of the metric, a natural problem in geometry as for the characterization of Einstein metrics [6]. The last section classifies Riemannian immersions which are critical points of $E_{2}$ with respect to isovolumetric variations.

See [20] for an account of biharmonic maps and [19] for an up-to-date bibliography.

Conventions. We work in the $C^{\infty}$ category, i.e. manifolds, metrics, connections and maps are smooth, and $\left(M^{m}, g\right)$ denotes a connected manifold of dimension $m$, without boundary, endowed with a Riemannian metric $g$. By an abuse of notation, $\langle$,$\rangle indicates inner products from different vec-$ tor bundles. The Riemann curvature operator is defined by $R(X, Y) Z=$ $\left[\nabla_{X}, \nabla_{Y}\right] Z-\nabla_{[X, Y]} Z$. The notations $\sharp$ and $b$ are for the standard musical isomorphisms. Compactness is not assumed unless explicitly stated. 


\section{The Euler-Lagrange Equation And applications}

For smooth maps $\phi:(M, g) \rightarrow(N, h)$ between Riemannian manifolds, $M$ compact and orientable, consider the bienergy functional $E_{2}$ :

$$
E_{2}: C^{\infty}(M, N) \rightarrow \mathbb{R}, \quad E_{2}(\phi)=\int_{M}|\tau(\phi)|^{2} v_{g},
$$

and, as mentioned in the introduction, a map is biharmonic if it is a critical point of $E_{2}$, that is, for any variation $\left\{\phi_{t}\right\}$ of $\phi,\left.\frac{d}{d t}\right|_{t=0} E_{2}\left(\phi_{t}\right)=0$.

Opting for a different angle of attack, one can vary the metric instead of the map, more precisely, given $\phi: M \rightarrow(N, h)$, consider the functional

$$
F: G \rightarrow \mathbb{R}, \quad F(g)=E_{2}(\phi),
$$

where $G$ is the set of Riemannian metrics on $M$. As $G$ is an infinite dimensional manifold ([7]), it admits a tangent space at $g$, the set of symmetric $(0,2)$-tensors on $M$, i.e.

$$
T_{g} G=C\left(\odot^{2} T^{*} M\right) .
$$

For a curve $t \rightarrow g_{t}$ in $G$ with $g_{0}=g$, denote by

$$
\omega=\left.\frac{d}{d t}\right|_{t=0}\left\{g_{t}\right\}=\delta\left(g_{t}\right) \in T_{g} G
$$

the corresponding variational tensor field which, in local coordinates, can be written

$$
\omega=\frac{\partial g_{i j}}{\partial t}(x, 0) d x^{i} d x^{j}=\omega_{i j} d x^{i} d x^{j},
$$

where $g_{t}=g_{i j}(x, t) d x^{i} d x^{j}$, and write $\delta=\left.\frac{d}{d t}\right|_{t=0}$ for the first variation.

For a one-parameter variation $\left\{g_{t}\right\}$ of $g$ we have

$$
F\left(g_{t}\right)=\frac{1}{2} \int_{M}\left|\tau_{t}(\phi)\right|^{2} v_{g_{t}} .
$$

We now compute $\delta\left(F\left(g_{t}\right)\right)$. Differentiating $F\left(g_{t}\right)$ leads to:

$$
\delta\left(F\left(g_{t}\right)\right)=\frac{1}{2} \int_{M} \delta\left(\left|\tau_{t}(\phi)\right|^{2}\right) v_{g}+\frac{1}{2} \int_{M}|\tau(\phi)|^{2} \delta\left(v_{g_{t}}\right) .
$$

The calculation of the first term breaks down in two lemmas.

Lemma 2.1. The vector field $\xi=(\operatorname{div} \omega)^{\sharp}-\frac{1}{2} \operatorname{grad}(\operatorname{trace} \omega)$ satisfies:

$$
\delta\left(\left|\tau_{t}(\phi)\right|^{2}\right)=-2\langle\tau(\phi) . \nabla d \phi, \omega\rangle-2\langle\tau(\phi), d \phi(\xi)\rangle,
$$

where $\tau(\phi) . \nabla d \phi \in C\left(\odot^{2} T^{*} M\right)$ is intended as

$$
(\tau(\phi) . \nabla d \phi)(X, Y)=\langle\tau(\phi), \nabla d \phi(X, Y)\rangle .
$$

Proof. In local coordinates $\left\{\left(U ; x^{i}\right)\right\}_{i=1}^{m}$ on $M$ and $\left\{\left(V ; y^{\alpha}\right)\right\}_{\alpha=1}^{n}$ on $N$ :

$$
\delta\left(\left|\tau_{t}(\phi)\right|^{2}\right)=\delta\left(h_{\alpha \beta}(\phi) \tau_{t}^{\alpha} \tau_{t}^{\beta}\right)=2 h_{\alpha \beta}\left(\delta\left(\tau_{t}^{\alpha}\right)\right) \tau^{\beta} .
$$


Now

$$
\begin{aligned}
\delta\left(\tau_{t}^{\alpha}\right) & =\delta\left(g^{i j}(x, t)\left(\frac{\partial^{2} \phi^{\alpha}}{\partial x^{i} \partial x^{j}}-\Gamma_{i j}^{k}(x, t) \phi_{k}^{\alpha}+{ }^{N} \Gamma_{\beta \sigma}^{\alpha} \phi_{i}^{\beta} \phi_{j}^{\sigma}\right)\right) \\
& =\left(\delta g^{i j}\right)(\nabla d \phi)_{i j}^{\alpha}-g^{i j}\left(\delta \Gamma_{i j}^{k}\right) \phi_{k}^{\alpha} .
\end{aligned}
$$

Since $g^{i l}(x, t) g_{l k}(x, t)=\delta_{k}^{i}, \delta g^{i j}=-g^{i a} g^{j b}\left(\delta g_{a b}\right)=-g^{i a} g^{j b} \omega_{a b}$, so

$$
\delta\left(\tau_{t}^{\alpha}\right)=-g^{i a} g^{j b} \omega_{a b}(\nabla d \phi)_{i j}^{\alpha}-g^{i j}\left(\delta \Gamma_{i j}^{k}\right) \phi_{k}^{\alpha} .
$$

Now we compute the term $g^{i j}\left(\delta \Gamma_{i j}^{k}\right) \phi_{k}^{\alpha}$. From

$$
\Gamma_{i j}^{k}(x, t)=\frac{1}{2} g^{k l}(x, t)\left(\frac{\partial g_{l i}}{\partial x^{j}}(x, t)+\frac{\partial g_{l j}}{\partial x^{i}}(x, t)-\frac{\partial g_{i j}}{\partial x^{l}}(x, t)\right)
$$

we have:

$$
\begin{aligned}
\delta \Gamma_{i j}^{k}= & \frac{1}{2}\left(\delta g^{k l}\right)\left(\frac{\partial g_{l i}}{\partial x^{j}}+\frac{\partial g_{l j}}{\partial x^{i}}-\frac{\partial g_{i j}}{\partial x^{l}}\right) \\
& +\frac{1}{2} g^{k l}\left(\frac{\partial^{2} g_{l i}}{\partial x^{j} \partial t}(x, 0)+\frac{\partial^{2} g_{l j}}{\partial x^{i} \partial t}(x, 0)-\frac{\partial^{2} g_{i j}}{\partial x^{l} \partial t}(x, 0)\right) \\
(2.4)= & -\frac{1}{2} g^{k a} g^{l b} \omega_{a b}\left(\frac{\partial g_{l i}}{\partial x^{j}}+\frac{\partial g_{l j}}{\partial x^{i}}-\frac{\partial g_{i j}}{\partial x^{l}}\right)+\frac{1}{2} g^{k l}\left(\frac{\partial \omega_{l i}}{\partial x^{j}}+\frac{\partial \omega_{l j}}{\partial x^{i}}-\frac{\partial \omega_{i j}}{\partial x^{l}}\right) \\
= & -g^{k a} \Gamma_{i j}^{b} \omega_{a b}+\frac{1}{2} g^{k l}\left(\frac{\partial \omega_{l i}}{\partial x^{j}}+\frac{\partial \omega_{l j}}{\partial x^{i}}-\frac{\partial \omega_{i j}}{\partial x^{l}}\right) .
\end{aligned}
$$

But

$$
\nabla_{j} \omega_{l i}=\frac{\partial \omega_{l i}}{\partial x^{j}}-\Gamma_{j l}^{h} \omega_{h i}-\Gamma_{j i}^{h} \omega_{l h}
$$

implies

$$
\frac{\partial \omega_{l i}}{\partial x^{j}}+\frac{\partial \omega_{l j}}{\partial x^{i}}-\frac{\partial \omega_{i j}}{\partial x^{l}}=\nabla_{j} \omega_{l i}+\nabla_{i} \omega_{l j}-\nabla_{l} \omega_{i j}+2 \Gamma_{i j}^{h} \omega_{h l} .
$$

So replacing in Equation (2.4) we obtain:

$$
\delta \Gamma_{i j}^{k}=\frac{1}{2} g^{k l}\left(\nabla_{j} \omega_{l i}+\nabla_{i} \omega_{l j}-\nabla_{l} \omega_{i j}\right)
$$

and

$$
g^{i j}\left(\delta \Gamma_{i j}^{k}\right) \phi_{k}^{\alpha}=g^{k l}\left(\nabla_{j} \omega_{l}^{j}-\frac{1}{2} \nabla_{l} \operatorname{trace} \omega\right) \phi_{k}^{\alpha}=\xi^{k} \phi_{k}^{\alpha} .
$$

From (2.2), (2.3) and (2.6), the lemma follows.

Lemma 2.2. Consider the one-form $d \phi . \tau(\phi) \in \Lambda^{1}(M)$ defined by $d \phi . \tau(\phi)(X)=$ $\langle d \phi(X), \tau(\phi)\rangle$, and sym $(\nabla(d \phi . \tau(\phi)))$ the symmetric part of $\nabla(d \phi . \tau(\phi))$, then:

$$
\int_{M}\langle\tau(\phi), d \phi(\xi)\rangle v_{g}=\int_{M}\left\langle-\operatorname{sym}(\nabla(d \phi \cdot \tau(\phi)))+\frac{1}{2}\left(\operatorname{div}(d \phi \cdot \tau(\phi))^{\sharp}\right) g, \omega\right\rangle v_{g} .
$$


Proof. First observe that:

$$
\langle\tau(\phi), d \phi(Z)\rangle=\left\langle d \phi . \tau(\phi), Z^{b}\right\rangle, \quad \forall Z \in C(T M) .
$$

By the definition of $\xi$

$$
\begin{aligned}
\int_{M}\langle\tau(\phi), d \phi(\xi)\rangle v_{g}= & \int_{M}\left\langle\tau(\phi), d \phi\left((\operatorname{div} \omega)^{\sharp}\right)\right\rangle v_{g} \\
& -\frac{1}{2} \int_{M}\langle\tau(\phi), d \phi(\operatorname{grad}(\operatorname{trace} \omega))\rangle v_{g}
\end{aligned}
$$

and, by (2.7), the first term on the right-hand side of (2.8) becomes

$$
\int_{M}\left\langle\tau(\phi), d \phi\left((\operatorname{div} \omega)^{\sharp}\right)\right\rangle v_{g}=\int_{M}\langle d \phi \cdot \tau(\phi), \operatorname{div} \omega\rangle v_{g} .
$$

On the other hand, if $\theta \in \Lambda^{1}(M), \sigma \in C\left(\odot^{2} T^{*} M\right)$, and $C(\theta, \sigma)=\left(\theta^{i} \sigma_{i j}\right) d x^{j}=$ $\left(\theta_{i} \sigma_{j}^{i}\right) d x^{j}$ denotes their contraction, we have:

$$
\langle\theta, \operatorname{div} \sigma\rangle=\operatorname{div}\left(C(\theta, \sigma)^{\sharp}\right)-\langle\operatorname{sym}(\nabla \theta), \sigma\rangle .
$$

Applying (2.9) to $\theta=d \phi . \tau(\phi)$ and $\sigma=\omega$, yields

$$
\int_{M}\left\langle\tau(\phi), d \phi\left((\operatorname{div} \omega)^{\sharp}\right)\right\rangle v_{g}=-\int_{M}\langle\operatorname{sym}(\nabla(d \phi . \tau(\phi))), \omega\rangle v_{g} .
$$

The second term on the right-hand side of (2.8) can then be written:

$$
\begin{aligned}
\int_{M}\langle\tau(\phi), d \phi(\operatorname{grad}(\operatorname{trace} \omega))\rangle v_{g} & =\int_{M}\langle d \phi \cdot \tau(\phi), d(\operatorname{trace} \omega)\rangle v_{g} \\
& =\int_{M}\left\langle(d \phi \cdot \tau(\phi))^{\sharp}, \operatorname{grad}(\operatorname{trace} \omega)\right\rangle v_{g} \\
& =-\int_{M}(\operatorname{trace} \omega) \operatorname{div}(d \phi \cdot \tau(\phi))^{\sharp} v_{g} \\
& =-\int_{M}\left\langle\left(\operatorname{div}(d \phi \cdot \tau(\phi))^{\sharp}\right) g, \omega\right\rangle v_{g} .
\end{aligned}
$$

The lemma follows from (2.8), (2.10) and (2.11).

This preparation is the key to:

Theorem 2.3. Let $\phi:(M, g) \rightarrow(N, h)$ be a smooth map, $M$ compact and orientable, and $\left\{g_{t}\right\}$ a one-parameter variation of $g$ through Riemannian metrics. Then

$$
\delta\left(F\left(g_{t}\right)\right)=-\frac{1}{2} \int_{M}\left\langle S_{2}, \omega\right\rangle v_{g},
$$

where $S_{2} \in C\left(\odot^{2} T^{*} M\right)$ is given by:

$$
\begin{aligned}
S_{2}(X, Y)= & \frac{1}{2}|\tau(\phi)|^{2}\langle X, Y\rangle+\langle d \phi, \nabla \tau(\phi)\rangle\langle X, Y\rangle \\
& -\left\langle d \phi(X), \nabla_{Y} \tau(\phi)\right\rangle-\left\langle d \phi(Y), \nabla_{X} \tau(\phi)\right\rangle .
\end{aligned}
$$


Proof. Recall that $\delta\left(v_{g_{t}}\right)=\left\langle\frac{1}{2} g, \omega\right\rangle v_{g}$ (see, for example, [24, 3]). Then, by Lemma 2.1 and 2.2. we can rewrite (2.1):

$$
\begin{aligned}
\delta\left(F\left(g_{t}\right)\right)=\int_{M} & \left\langle\left[\frac{1}{4}|\tau(\phi)|^{2} g-\frac{1}{2}\left(\operatorname{div}(d \phi \cdot \tau(\phi))^{\sharp}\right) g\right.\right. \\
& +\operatorname{sym}(\nabla(d \phi \cdot \tau(\phi)))-\tau(\phi) . \nabla d \phi], \omega\rangle v_{g} .
\end{aligned}
$$

The formula (cf. [15])

$$
\operatorname{div}(d \phi . \tau(\phi))^{\sharp}=|\tau(\phi)|^{2}+\langle d \phi, \nabla \tau(\phi)\rangle
$$

and the expression of $\operatorname{sym}(\nabla(d \phi . \tau(\phi)))$ :

$$
\begin{aligned}
\operatorname{sym}(\nabla(d \phi . \tau(\phi)))(X, Y)= & \frac{1}{2}((\nabla(d \phi . \tau(\phi)))(X, Y)+(\nabla(d \phi . \tau(\phi)))(Y, X)) \\
= & \langle\nabla d \phi(X, Y), \tau(\phi)\rangle \\
& +\frac{1}{2}\left(\left\langle d \phi(X), \nabla_{Y} \tau(\phi)\right\rangle+\left\langle d \phi(Y), \nabla_{X} \tau(\phi)\right\rangle\right),
\end{aligned}
$$

end the proof.

As mentioned in the introduction, $S_{2}$ was known by Jiang in [18, where he proved the following

Theorem 2.4 (18]). For any map $\phi:(M, g) \rightarrow(N, h)$ :

$$
\operatorname{div} S_{2}(Y)=-\left\langle\tau_{2}(\phi), d \phi(Y)\right\rangle, \quad \forall Y \in C(T M) .
$$

Proof. We give a proof for the sake of completeness. Write $S_{2}=T_{1}+T_{2}$, where $T_{1}, T_{2} \in C\left(\odot^{2} T^{*} M\right)$ are defined by

$$
\begin{aligned}
& T_{1}(X, Y)=\frac{1}{2}|\tau(\phi)|^{2}\langle X, Y\rangle+\langle d \phi, \nabla \tau(\phi)\rangle\langle X, Y\rangle \\
& T_{2}(X, Y)=-\left\langle d \phi(X), \nabla_{Y} \tau(\phi)\right\rangle-\left\langle d \phi(Y), \nabla_{X} \tau(\phi)\right\rangle .
\end{aligned}
$$

Let $p \in M$ and $\left\{X_{i}\right\}_{i=1}^{m}$ a geodesic frame centered on $p$. Writing $Y=Y^{i} X_{i}$, at $p$, we have:

$$
\begin{aligned}
\operatorname{div} T_{1}(Y)= & \sum_{i}\left(\nabla_{X_{i}} T_{1}\right)\left(X_{i}, Y\right)=\sum_{i}\left(X_{i}\left(T_{1}\left(X_{i}, Y\right)\right)-T_{1}\left(X_{i}, \nabla_{X_{i}} Y\right)\right) \\
= & \sum_{i}\left(X_{i}\left(\frac{1}{2}|\tau(\phi)|^{2} Y^{i}+\sum_{j}\left\langle d \phi\left(X_{j}\right), \nabla_{X_{j}} \tau(\phi)\right\rangle Y^{i}\right)\right. \\
(2.14) \quad & \left.-\frac{1}{2}|\tau(\phi)|^{2} X_{i} Y^{i}-\sum_{j}\left\langle d \phi\left(X_{j}\right), \nabla_{X_{j}} \tau(\phi)\right\rangle\left(X_{i} Y^{i}\right)\right) \\
= & \left\langle\nabla_{Y} \tau(\phi), \tau(\phi)\right\rangle+\sum_{i}\left\langle\nabla d \phi\left(Y, X_{i}\right), \nabla_{X_{i}} \tau(\phi)\right\rangle \\
& +\sum_{i}\left\langle d \phi\left(X_{i}\right), \nabla_{Y} \nabla_{X_{i}} \tau(\phi)\right\rangle \\
= & \left\langle\nabla_{Y} \tau(\phi), \tau(\phi)\right\rangle+\operatorname{trace}\langle\nabla d \phi(Y, \cdot), \nabla \cdot \tau(\phi)\rangle \\
& +\operatorname{trace}\left\langle d \phi(\cdot), \nabla^{2} \tau(\phi)(Y, \cdot)\right\rangle,
\end{aligned}
$$


whilst

$$
\begin{aligned}
\operatorname{div} T_{2}(Y)= & \sum_{i}\left(X_{i}\left(T_{2}\left(X_{i}, Y\right)\right)-T_{2}\left(X_{i}, \nabla_{X_{i}} Y\right)\right) \\
= & -\left\langle\nabla_{Y} \tau(\phi), \tau(\phi)\right\rangle-\sum_{i}\left\langle\nabla d \phi\left(Y, X_{i}\right), \nabla_{X_{i}} \tau(\phi)\right\rangle \\
& -\sum_{i}\left\langle d \phi\left(X_{i}\right), \nabla_{X_{i}} \nabla_{Y} \tau(\phi)-\nabla_{\nabla_{X_{i}}} \tau(\phi)\right\rangle \\
& +\langle d \phi(Y), \Delta \tau(\phi)\rangle \\
= & -\left\langle\nabla_{Y} \tau(\phi), \tau(\phi)\right\rangle-\operatorname{trace}\langle\nabla d \phi(Y, \cdot), \nabla \cdot \tau(\phi)\rangle \\
& -\operatorname{trace}\left\langle d \phi(\cdot), \nabla^{2} \tau(\phi)(\cdot, Y)\right\rangle+\langle d \phi(Y), \Delta \tau(\phi)\rangle .
\end{aligned}
$$

Summing (2.14) and (2.15) gives:

$$
\begin{aligned}
\operatorname{div} S_{2}(Y) & =\langle d \phi(Y), \Delta \tau(\phi)\rangle+\sum_{i}\left\langle d \phi\left(X_{i}\right), R\left(Y, X_{i}\right) \tau(\phi)\right\rangle \\
& =-\left\langle\tau_{2}(\phi), d \phi(Y)\right\rangle .
\end{aligned}
$$

Theorem 2.4 links $S_{2}$ with the bitension field and immediately leads to the following characterizations.

Corollary 2.5. Let $\phi:(M, g) \rightarrow(N, h)$ be a smooth map.

a) If $\phi$ is a Riemannian immersion, then $\operatorname{div} S_{2}=0$ if and only if the tangent part of $\tau_{2}(\phi)$ vanishes.

b) If $\phi$ is a submersion (not necessarily Riemannian), then $\operatorname{div} S_{2}=0$ if and only if $\phi$ is biharmonic.

These results are applied to construct proper (i.e. non-harmonic) biharmonic maps.

Proposition 2.6. Let $\phi:(M, g) \rightarrow(N, h)$ be a submersion, $M$ noncompact. Assume that $\tau(\phi)$ is basic, i.e. $\tau(\phi)=\zeta \circ \phi$, where $\zeta \in C(T N)$. If $\zeta$ is Killing and $|\zeta|^{2}=c^{2} \neq 0$ is constant, then $\phi$ is proper biharmonic.

Proof. We prove that $\operatorname{div} S_{2}=0$. The tensor $S_{2}$ takes, in this case, the expression:

$S_{2}(X, Y)=\left\{\frac{c^{2}}{2}+\langle d \phi, \nabla \tau(\phi)\rangle\right\}\langle X, Y\rangle-\left\langle d \phi(X), \nabla_{Y} \tau(\phi)\right\rangle-\left\langle d \phi(Y), \nabla_{X} \tau(\phi)\right\rangle$.

Now, let $p$ be a point in $M$ and $\left\{X_{i}\right\}_{i=1}^{m}$ an orthonormal basis of $T_{p} M$ such that $\left\{X_{\alpha}\right\}_{\alpha=1}^{n}$ belongs to $T_{p}^{H} M=\left(T_{p}^{V} M\right)^{\perp}$ and $\left\{X_{a}\right\}_{a=n+1}^{m}$ to $T_{p}^{V} M=$ ker $d \phi(p)$. As $\zeta$ is Killing:

$$
\begin{aligned}
\langle d \phi, \nabla \tau(\phi)\rangle(p) & =\sum_{\alpha}\left\langle d \phi_{p}\left(X_{\alpha}\right), \nabla_{X_{\alpha}} \tau(\phi)\right\rangle+\sum_{a}\left\langle d \phi_{p}\left(X_{a}\right), \nabla_{X_{a}} \tau(\phi)\right\rangle \\
& =\sum_{\alpha}\left\langle d \phi_{p}\left(X_{\alpha}\right), \nabla_{d \phi_{p}\left(X_{\alpha}\right)}^{N} \zeta\right\rangle \\
& =0
\end{aligned}
$$


and, for any $X, Y \in T_{p} M$,

$$
\begin{aligned}
S_{2}(p)(X, Y) & =\frac{c^{2}}{2}\langle X, Y\rangle-\left(\left\langle d \phi_{p}(X), \nabla_{d \phi_{p}(Y)}^{N} \zeta\right\rangle+\left\langle d \phi_{p}(Y), \nabla_{d \phi_{p}(X)}^{N} \zeta\right\rangle\right) \\
& =\frac{c^{2}}{2}\langle X, Y\rangle .
\end{aligned}
$$

Thus $S_{2}=\frac{c^{2}}{2} g$ is divergence free.

If $M$ is compact, since $\langle d \phi, \nabla \tau(\phi)\rangle=0$, integrating (2.13), yields $\tau(\phi)=$ 0 .

We apply Proposition 2.6 to three situations. Recall that a function is affine if its restriction to any geodesic is affine with respect to the real parameter, or, equivalently, if its gradient is parallel ([23]).

Application 2.7. Let $(M, g)$ and $(N, h)$ be Riemannian manifolds and denote by $M \times_{f^{2}} N$ their warped product with respect to a positive function on $M$, then the projection $\pi: M \times_{f^{2}} N \rightarrow M$ is a Riemannian submersion with $\tau(\pi)=n \operatorname{grad}(\ln f) \circ \pi$. When $\ln f$ is affine, $\operatorname{grad}(\ln f)$ is Killing of constant norm ([5]), hence $\pi$ is biharmonic.

Application 2.8. For a vector field $\zeta$ on $M, T M$ can be endowed with a Sasaki-type metric such that the projection $\pi: T M \rightarrow M$ is a Riemannian submersion and $\tau(\pi)=-(m+1) \zeta \circ \pi([21])$. Choosing $\zeta$ Killing of constant norm makes $\pi$ biharmonic.

In Application 2.7, the fibres of $\pi$ do not have the same volume, otherwise the next proposition would force the map to be harmonic.

Proposition 2.9. Let $\phi:(M, g) \rightarrow(N, h)$ be a Riemannian submersion with basic tension field, $M$ compact and orientable. If $\operatorname{Vol}\left(\phi^{-1}(q)\right)$ is constant then $\phi$ is harmonic.

Proof. Write $\tau(\phi)=\zeta \circ \phi, \zeta \in C(T N)$ then, by a simple calculation, $\langle d \phi, \nabla \tau(\phi)\rangle=(\operatorname{div} \zeta) \circ \phi$. If $\operatorname{Vol}\left(\phi^{-1}(q)\right)=c$ for all $q \in N$, the coarea formula $(\underline{8}, 9])$, would impose:

$$
\begin{aligned}
\int_{M}\langle d \phi, \nabla \tau(\phi)\rangle v_{g} & =\int_{M}(\operatorname{div} \zeta) \circ \phi v_{g} \\
& =\int_{N}\left(\int_{\phi^{-1}(q)}(\operatorname{div} \zeta)(q) v_{g_{q}}\right) v_{h}=c \int_{N} \operatorname{div} \zeta v_{h}=0,
\end{aligned}
$$

where $g_{q}$ is the induced metric on $\phi^{-1}(q) \subset M$. On the other hand,

$$
\int_{M}\langle d \phi, \nabla \tau(\phi)\rangle v_{g}=-\int_{M}|\tau(\phi)|^{2} v_{g}=-\int_{M}|\zeta|^{2} \circ \phi v_{g}=-c \int_{N}|\zeta|^{2} v_{h} .
$$

Application 2.10. A submersion $\phi:(M, g) \rightarrow(N, h)$ is horizontally conformal ([3] ) if there exists a positive function $\lambda \in C(M)$, the dilation, such that

$$
h\left(d \phi_{p}(X), d \phi_{p}(Y)\right)=\lambda^{2} g_{p}(X, Y), \quad \forall X, Y \in\left(\operatorname{ker} d \phi_{p}\right)^{\perp} .
$$


A partial biharmonic analogue of the Baird-Eells Theorem, which states that, for horizontally homothetic submersion harmonicity is equivalent to minimality of the fibres, would be:

Proposition 2.11. Let $\phi:(M, g) \rightarrow(N, h)$ be a submersion with dilation $\lambda=\tilde{\lambda} \circ \phi$ and minimal fibers. If $\tilde{\lambda}^{2}$ is non-constant and affine, and $n \neq 2$, then $\phi$ is proper biharmonic.

Proof. By a straightforward computation we get $\tau(\phi)=\frac{2-n}{2}\left(\operatorname{grad} \tilde{\lambda}^{2}\right) \circ \phi$ and, since $\tilde{\lambda}^{2}$ is affine, then grad $\tilde{\lambda}^{2}$ is Killing of constant norm.

We point out that a horizontally conformal submersion $\phi:(M, g) \rightarrow$ $(N, h)$, with constant dilation along the fibres, is the composition between a Riemannian submersion $\varphi:(M, g) \rightarrow(N, \bar{h})$ and the identity map 1 : $(N, \bar{h}) \rightarrow(N, h)$, where $\bar{h}$ is conformal to $h$.

Proposition 2.12. Let $1:\left(M^{m}, g\right) \rightarrow\left(M^{m}, \bar{g}=e^{2 \rho} g\right)$ be the identity map, $m \neq 2$ and $\rho \in C^{\infty}(M)$.

a) If $\rho=\ln \sqrt{f}, f$ a nonconstant affine positive function on $(M, \bar{g})$, then 1 is proper biharmonic.

b) If $\rho$ is a nonconstant affine function on $(M, \bar{g})$, then $\mathbf{1}$ is proper biharmonic if and only if $m=4$.

Proof. a) To prove $\operatorname{div} S_{2}=0$, we compute:

$$
\tau(\mathbf{1})=(2-m) \operatorname{grad}_{g} \rho=(2-m) e^{2 \rho} \operatorname{grad}_{\bar{g}} \rho=\frac{2-m}{2} \operatorname{grad}_{\bar{g}}\left(e^{2 \rho}\right) .
$$

By hypothesis, $e^{2 \rho}=f$ is affine on $(M, \bar{g})$, thus $\operatorname{grad}_{\bar{g}}\left(e^{2 \rho}\right)$ is parallel and $\bar{\nabla} \tau(\mathbf{1})=0, \bar{\nabla}$ being the connection of $\bar{g}$. Therefore $S_{2}=\frac{1}{2} c^{2} g$, with $c^{2}=$ $|\tau(\mathbf{1})| \frac{2}{g}$ constant.

We note that this result can be also obtained by Proposition 2.11

b) From $\bar{\nabla} \operatorname{grad}_{\bar{g}} \rho=0$ we infer:

$$
|\tau(\mathbf{1})| \frac{2}{g}=(2-m)^{2} c^{2} e^{4 \rho}, \quad \bar{\nabla}_{X} \tau(\mathbf{1})=2(2-m) e^{2 \rho}(X \rho) \operatorname{grad}_{\bar{g}} \rho,
$$

where $c^{2}=\left|\operatorname{grad}_{\bar{g}} \rho\right| \frac{2}{g}$ is constant. Put $S_{2}=T_{1}-T_{2}$, with

$$
T_{1}=\left(\frac{1}{2}|\tau(\mathbf{1})|^{2}+\langle d \mathbf{1}, \nabla \tau(\mathbf{1})\rangle\right) g=\frac{(2-m)(6-m)}{2} c^{2} e^{4 \rho} g
$$

and

$$
T_{2}(X, Y)=\left\langle d \mathbf{1}(X), \nabla_{Y} \tau(\mathbf{1})\right\rangle+\left\langle d \mathbf{1}(Y), \nabla_{X} \tau(\mathbf{1})\right\rangle=4(2-m) e^{2 \rho}(X \rho)(Y \rho) .
$$

Then:

$$
\begin{aligned}
\operatorname{div} T_{1}(Z) & =\frac{(2-m)(6-m)}{2} c^{2} g\left(\operatorname{grad} e^{4 \rho}, Z\right) \\
& =2(2-m)(6-m) c^{2} e^{4 \rho}(Z \rho),
\end{aligned}
$$


and

$$
\begin{aligned}
\operatorname{div} T_{2}(Z)= & 8(2-m) c^{2} e^{4 \rho}(Z \rho)-4(2-m) e^{2 \rho}(\Delta \rho)(Z \rho) \\
& +4(2-m) e^{2 \rho} \operatorname{Hess} \rho\left(\operatorname{grad}_{g} \rho, Z\right) .
\end{aligned}
$$

If we look at $\rho$ as $\bar{\rho} \circ \mathbf{1}$, where $\bar{\rho}:(M, \bar{g}) \rightarrow \mathbb{R}$, and apply the chain rule, we get

$$
-\Delta \rho=(2-m) c^{2} e^{2 \rho}, \quad \operatorname{Hess} \rho\left(\operatorname{grad}_{g} \rho, Z\right)=c^{2} e^{2 \rho}(Z \rho) .
$$

Replace (2.18) in (2.17) to obtain:

$$
\operatorname{div} T_{2}(Z)=4(2-m)(5-m) c^{2} e^{4 \rho}(Z \rho),
$$

and from (2.16) and (2.19)

$$
\operatorname{div} S_{2}(Z)=2(2-m)(m-4) c^{2} e^{4 \rho}(Z \rho) .
$$

We conclude since $\rho$ is not constant and $m \neq 2$.

Remark 2.13. The function $\rho$ in Proposition 2.12 is isoparametric (though not affine) on $(M, g)$. On Einstein manifolds, interesting links between biharmonicity of the identity (modulo a conformal change of the metric) and isoparametricity of the conformal factor have been discovered in [2, 4].

We close this section with another application of $\operatorname{div} S_{2}=0$.

Let $\sigma \in C\left(\odot^{2} T^{*} M\right), \sigma=\sigma_{i j} d x^{i} d x^{j}$, and $X \in C(T M), X=X^{i} \frac{\partial}{\partial x^{i}}$. Consider the contraction $C(X, \sigma)=X^{j} g^{i h} \sigma_{h j} \frac{\partial}{\partial x^{i}}=X^{j} \sigma_{j}^{i} \frac{\partial}{\partial x^{i}}=(\sigma(X, \cdot))^{\sharp}$. As

$$
\operatorname{div} C(X, \sigma)=\operatorname{div} \sigma(X)+\frac{1}{2}\left\langle\sigma, L_{X} g\right\rangle
$$

we deduce:

Proposition 2.14. If $X$ is Killing and $\phi:(M, g) \rightarrow(N, h)$ biharmonic map, then $C\left(X, S_{2}\right)$ is divergence free.

\section{VANISHING OF THE BIHARMONIC STRESS-ENERGY TENSOR}

Clearly, from (2.12), harmonic implies $S_{2}=0$, so it is only natural to study the converse. Note that $F(g)$ is nonnegative and zero if and only if $\phi$ is harmonic. Thus our quest is of critical points $\left(S_{2}=0\right)$ which are minima.

Before embarking on this problem, observe that $S_{2}=0$ does not, in general, imply harmonicity, as illustrated by the non-geodesic cubic curve $\gamma(t)=t^{3} a, a \in \mathbb{R}^{n}$. Yet, if we impose arc-length parametrization, we have:

Proposition 3.1. Let $\gamma: I \subset \mathbb{R} \rightarrow(N, h)$ be a curve parametrized by arc-length, assume $S_{2}=0$, then $\gamma$ is geodesic.

Proof. A direct computation shows:

$$
\begin{aligned}
0 & =S_{2}\left(\frac{\partial}{\partial t}, \frac{\partial}{\partial t}\right)=\frac{1}{2}|\tau(\gamma)|^{2}-\left\langle d \gamma\left(\frac{\partial}{\partial t}\right), \nabla_{\frac{\partial}{\partial t}} \tau(\gamma)\right\rangle \\
& =\frac{3}{2}|\tau(\gamma)|^{2}
\end{aligned}
$$


When the domain is a surface, $S_{2}=0$ is indeed very strong.

Proposition 3.2. Let $\phi:\left(M^{2}, g\right) \rightarrow(N, h)$ be a map from a surface, then $S_{2}=0$ implies $\phi$ harmonic.

Proof. The trace of $S_{2}$ gives:

$$
\begin{aligned}
0 & =\operatorname{trace} S_{2}=|\tau(\phi)|^{2}+2\langle d \tau(\phi), d \phi\rangle-2\langle d \tau(\phi), d \phi\rangle \\
& =|\tau(\phi)|^{2} .
\end{aligned}
$$

For the sequel, we first need a reformulation of $S_{2}=0$ :

Proposition 3.3. Let $\phi:(M, g) \rightarrow(N, h), m \neq 2$, then $S_{2}=0$ if and only if

$$
\frac{1}{m-2}|\tau(\phi)|^{2}\langle X, Y\rangle+\left\langle\nabla_{X} \tau(\phi), d \phi(Y)\right\rangle+\left\langle\nabla_{Y} \tau(\phi), d \phi(X)\right\rangle=0,
$$

$\forall X, Y \in C(T M)$.

Proposition 3.4. A map $\phi:\left(M^{m}, g\right) \rightarrow(N, h), m>2$, with $S_{2}=0$ and rank $\phi \leq m-1$ is harmonic.

Proof. Take $p \in M$, as $\operatorname{rank} \phi(p) \leq m-1$, there exists a unit vector $X_{p} \in$ ker $d \phi_{p}$ and for $X=Y=X_{p}$, (3.1) becomes $\tau(\phi)(p)=0$.

Corollary 3.5. Let $\phi:(M, g) \rightarrow(N, h)$ be a submersion $(m>n)$, if $S_{2}=0$ then $\phi$ is harmonic.

Recall the following result, originally due to Jiang

Theorem 3.6 (18]). A map $\phi:(M, g) \rightarrow(N, h), m \neq 4$, with $S_{2}=0, M$ compact and orientable, is harmonic.

Proof. Trace of $S_{2}$ is

$$
0=\operatorname{trace} S_{2}=\frac{m}{2}|\tau(\phi)|^{2}+m\langle d \tau(\phi), d \phi\rangle-2\langle d \tau(\phi), d \phi\rangle,
$$

and integrating over $M$ :

$$
0=\frac{4-m}{2} \int_{M}|\tau(\phi)|^{2} v_{g}
$$

hence, as $m \neq 4, \phi$ is harmonic.

Remark 3.7. An alternative proof is: consider the one-parameter variation $\left\{g_{t}=(1+t) g\right\}$, then

$$
F\left(g_{t}\right)=(1+t)^{\frac{m-4}{2}} F(g) .
$$

Now $S_{2}=0$ implies $\delta\left(F\left(g_{t}\right)\right)=0$ hence $(m \neq 4), F(g)=0$, i.e. $\phi$ is harmonic.

When $M$ is not necessarily compact, Theorem 3.6 can be rewritten for Riemannian immersions: 
Proposition 3.8. A Riemannian immersion $\phi:(M, g) \rightarrow(N, h)$ with $S_{2}=$ $0, m \neq 4$, is minimal.

Proof. By (2.13), $\langle d \tau(\phi), d \phi\rangle=-|\tau(\phi)|^{2}$, and replacing in (3.2) yields

$$
\frac{4-m}{2}|\tau(\phi)|^{2}=0
$$

forcing $\phi$ to be minimal.

The next result introduces integral conditions ensuring that $S_{2}=0$ reveals harmonicity. First we cite Yau's version of Stokes Theorem:

Lemma $3.9(25])$. Let $\left(M^{m}, g\right)$ be a complete Riemannian manifold and $\omega$ a smooth integrable $(m-1)$-form defined on $M$. Then there exists a sequence of domains $B_{i}$ in $M$ such that $M=\bigcup_{i} B_{i}, B_{i} \subset B_{i+1}$, and $\lim _{i \rightarrow \infty} \int_{B_{i}} d \omega=$ 0 .

Theorem 3.10. Let $(M, g), m \neq 4$, be an orientable complete Riemannian manifold and $\phi:(M, g) \rightarrow(N, h)$ a map with $S_{2}=0$. If $\int_{M}|d \phi . \tau(\phi)| v_{g}<$ $\infty$ then $\phi$ is harmonic.

Proof. For $m=2$, this is Proposition 3.2, so now assume $m \neq 2$. The trace of $S_{2}$ and (2.13) result in:

$$
\frac{m-4}{2(m-2)}|\tau(\phi)|^{2} v_{g}=(\operatorname{div} X) v_{g}=d\left(i_{X} v_{g}\right)
$$

where $X=(d \phi . \tau(\phi))^{\sharp}$. We now apply Lemma 3.9 to $\omega=i_{X} v_{g}$. To compute the norm of $\omega$, choose $p \in M$ and a local normal chart $\left(U ; x^{k}\right)_{k=1}^{m}$ around it:

$$
v_{g}(p)=d x^{1} \wedge \ldots \wedge d x^{m}, \quad\left(i_{X(p)} v_{g}\right)_{i \ldots \hat{k} \ldots m}=(-1)^{k+1} \xi^{k},
$$

so

$$
|\omega|^{2}(p)=\left|i_{X} v_{g}\right|^{2}(p)=\sum_{i_{1}, \ldots, i_{m-1}=1}^{m}\left(\left(i_{X(p)} v_{g}\right)_{i_{1}, \ldots, i_{m-1}}\right)^{2}=(m-1) !|X|^{2}(p) .
$$

Now, $\int_{M}|X| v_{g}=\int_{M}|d \phi . \tau(\phi)|<\infty$, so $\omega$ is integrable. By Lemma 3.9. $\lim _{i \rightarrow \infty} \int_{B_{i}} d \omega v_{g}=\frac{m-4}{2(m-2)} \lim _{i \rightarrow \infty} \int_{B_{i}}|\tau(\phi)|^{2} v_{g}=0$, hence $\phi$ is harmonic.

Corollary 3.11. Let $(M, g), m \neq 4$, be an orientable complete Riemannian manifold and $\phi:(M, g) \rightarrow(N, h)$ a map with finite energy and bienergy. If $S_{2}=0$ then $\phi$ is harmonic.

Proof. Let $p$ be a point of $M$ and $\left(U ; x^{i}\right)_{i=1}^{m},\left(V ; y^{\alpha}\right)_{\alpha=1}^{n}$ be local normal charts around $p$ and $\phi(p)$, respectively. At $p$

$$
\left\langle d \phi\left(\frac{\partial}{\partial x^{i}}\right), \tau(\phi)\right\rangle=\left\langle\phi_{i}^{\alpha} \frac{\partial}{\partial y^{\alpha}}, \tau(\phi)^{\beta} \frac{\partial}{\partial y^{\beta}}\right\rangle=\sum_{\alpha} \phi_{i}^{\alpha} \tau(\phi)^{\alpha},
$$


so, by Cauchy Inequality

$$
\left(\left\langle d \phi\left(\frac{\partial}{\partial x^{i}}\right), \tau(\phi)\right\rangle\right)^{2} \leq\left(\sum_{\alpha}\left(\phi_{i}^{\alpha}\right)^{2}\right)|\tau(\phi)|^{2} .
$$

Therefore, at $p$

$$
|d \phi . \tau(\phi)|^{2}=\sum_{i}\left(\left\langle d \phi\left(\frac{\partial}{\partial x^{i}}\right), \tau(\phi)\right\rangle\right)^{2} \leq|d \phi|^{2}|\tau(\phi)|^{2} .
$$

Consequently,

$\int_{M}|d \phi . \tau(\phi)| v_{g} \leq \int_{M}|d \phi||\tau(\phi)| v_{g} \leq\left(\int_{M}|d \phi|^{2} v_{g}\right)^{\frac{1}{2}}\left(\int_{M}|\tau(\phi)|^{2} v_{g}\right)^{\frac{1}{2}}<\infty$, and we can apply Theorem 3.10 .

When $m=4$, the situation is drastically different, that is $S_{2}=0$ does not always imply $\phi$ harmonic, even if $M$ is compact.

Theorem 3.12 ([18]). A non-minimal Riemannian immersion $\phi:\left(M^{4}, g\right) \rightarrow$ $(N, h)$ satisfies $S_{2}=0$ if and only if it is pseudo-umbilical.

Proof. First note that for a Riemannian immersion, $S_{2}$ reduces to

$$
S_{2}(X, Y)=-\frac{1}{2}|\tau(\phi)|^{2}\langle X, Y\rangle+2\langle\tau(\phi), B(X, Y)\rangle,
$$

$B=\nabla d \phi$ being its second fundamental form. Recall that a Riemannian immersion is pseudo-umbilical if and only if its shape operator $A$ satisfies:

$$
A_{\tau(\phi)}=\frac{1}{m}|\tau(\phi)|^{2} I
$$

equivalently

$$
\langle B(X, Y), \tau(\phi)\rangle=\frac{1}{m}|\tau(\phi)|^{2}\langle X, Y\rangle .
$$

Comparing with (3.3) ends the proof.

To weaken hypotheses, we consider conformal immersions and show "rigidity".

Proposition 3.13. Let $\phi:\left(M^{4}, g=e^{2 \rho} \phi^{*} h\right) \rightarrow(N, h)$ be a conformal immersion, $M$ compact and orientable. If $S_{2}=0$ then $\rho$ is constant and $\bar{\phi}:\left(M^{4}, \phi^{*} h\right) \rightarrow(N, h)$ is a pseudo-umbilical Riemannian immersion.

Proof. Put $\bar{g}=e^{-2 \rho} g=\phi^{*} h$, denote by $\bar{\nabla}$ the connection of $\bar{g}$, by $\mathbf{1}$ : $(M, g) \rightarrow(M, \bar{g})$ the identity map, so that $\bar{\phi}:(M, \bar{g}) \rightarrow(N, h)$ is a Riemannian immersion, and $\phi=\bar{\phi} \circ \mathbf{1}$. By chain rule:

$$
\begin{aligned}
\tau(\phi) & =d \bar{\phi}(\tau(\mathbf{1}))+\operatorname{trace}_{g} \nabla d \bar{\phi}(d \mathbf{1} \cdot d \mathbf{1} \cdot) \\
& =2 d \bar{\phi}\left(\operatorname{grad}_{g} \rho\right)+e^{-2 \rho} \tau(\bar{\phi}),
\end{aligned}
$$

and computing the norm yields

$$
\begin{aligned}
|\tau(\phi)|^{2} & =4\left|d \bar{\phi}\left(\operatorname{grad}_{g} \rho\right)\right|^{2}+e^{-4 \rho}|\tau(\bar{\phi})|^{2} \\
& =4 e^{-2 \rho}\left|\operatorname{grad}_{g} \rho\right|_{g}^{2}+e^{-4 \rho}|\tau(\bar{\phi})|^{2} .
\end{aligned}
$$


Next:

$\left\langle\nabla_{X} d \bar{\phi}\left(\operatorname{grad}_{g} \rho\right), d \bar{\phi}(Y)\right\rangle=\left\langle\nabla d \bar{\phi}\left(X, \operatorname{grad}_{g} \rho\right)+d \bar{\phi}\left(\bar{\nabla}_{X} \operatorname{grad}_{g} \rho\right), d \bar{\phi}(Y)\right\rangle$

$$
\begin{aligned}
& =\bar{g}\left(\bar{\nabla}_{X} \operatorname{grad}_{g} \rho, Y\right)=\bar{g}\left(\bar{\nabla}_{X}\left(e^{-2 \rho} \operatorname{grad}_{\bar{g}} \rho\right), Y\right) \\
& =-2 e^{-2 \rho}(X \rho)(Y \rho)+e^{-2 \rho} \bar{g}\left(\bar{\nabla}_{X} \operatorname{grad}_{\bar{g}} \rho, Y\right),
\end{aligned}
$$

whilst

$$
\left\langle\nabla_{X} e^{-2 \rho} \tau(\bar{\phi}), d \phi(Y)\right\rangle=-e^{-2 \rho}\langle\tau(\bar{\phi}), \nabla d \bar{\phi}(X, Y)\rangle .
$$

From (3.5) and (3.6), we obtain

$$
\begin{aligned}
\left\langle\nabla_{X} \tau(\phi), d \phi(Y)\right\rangle=e^{-2 \rho} & -4(X \rho)(Y \rho)+2 \bar{g}\left(\bar{\nabla}_{X} \operatorname{grad}_{\bar{g}} \rho, Y\right) \\
& -\langle\tau(\bar{\phi}), \nabla d \bar{\phi}(X, Y)\rangle)
\end{aligned}
$$

and

$$
\langle d \phi, \nabla \tau(\phi)\rangle=-e^{-4 \rho}\left(4\left|\operatorname{grad}_{\bar{g}} \rho\right| \frac{2}{g}+2 \Delta_{\bar{g}} \rho+|\tau(\bar{\phi})|^{2}\right),
$$

where $\Delta_{\bar{g}}$ is the Laplacian with respect to $\bar{g}$.

Let $\overline{S_{2}}$ be the stress-energy tensor of $\bar{\phi}$, by (3.4), (3.7), (3.8), and $\left|\operatorname{grad}_{g} \rho\right|_{g}^{2}=$ $e^{-2 \rho}\left|\operatorname{grad}_{\bar{g}} \rho\right| \frac{2}{g}$, a straightforward computation gives:

$$
\begin{aligned}
e^{2 \rho} S_{2}(X, Y)= & \overline{S_{2}}(X, Y)-2\left(|\operatorname{grad} \bar{g} \rho| \frac{2}{g}+\Delta \bar{g} \rho\right) \bar{g}(X, Y) \\
(3.9) & +8(X \rho)(Y \rho)-2\left(\bar{g}\left(\bar{\nabla}_{X} \operatorname{grad}_{\bar{g}} \rho, Y\right)+\bar{g}\left(\bar{\nabla}_{Y} \operatorname{grad} \bar{g} \rho, X\right)\right) .
\end{aligned}
$$

Assume $S_{2}=0$, taking the $\bar{g}$-trace of (3.9), we obtain $\Delta \bar{g} \rho=0$, hence, as $M$ is compact, $\rho$ is constant. Replacing in (3.9) shows that $\overline{S_{2}}$ vanishes as well.

Proposition 3.14. Let $\phi:\left(M^{4}, g\right) \rightarrow(N, h)$ be a map, $M$ compact, with $S_{2}=0$. If there exists $\rho \in C^{\infty}(M)$ such that $\bar{\phi}:\left(M^{4}, \bar{g}=e^{2 \rho} g\right) \rightarrow(N, h)$ is harmonic, then $\phi$ is harmonic. Moreover, if rank $\phi=4, \rho$ must be constant.

Proof. Denote by $1:(M, g) \rightarrow(M, \bar{g})$ the identity map, then $\tau(\phi)=$ $-2 d \bar{\phi}\left(\operatorname{grad}_{g} \rho\right)$. Choosing $X=Y=\operatorname{grad}_{g} \rho$ in (3.1), implies:

$$
\begin{aligned}
0 & =\frac{1}{2}|\tau(\phi)|^{2}\left|\operatorname{grad}_{g} \rho\right|_{g}^{2}+2\left\langle\nabla_{\operatorname{grad}_{g} \rho} \tau(\phi), d \phi\left(\operatorname{grad}_{g} \rho\right)\right\rangle \\
& =\frac{1}{2}|\tau(\phi)|^{2}\left|\operatorname{grad}_{g} \rho\right|_{g}^{2}-\left\langle\nabla_{\operatorname{grad}_{g} \rho} \tau(\phi), \tau(\phi)\right\rangle \\
& =\frac{1}{2}|\tau(\phi)|^{2}\left|\operatorname{grad}_{g} \rho\right|_{g}^{2}-\frac{1}{2} \operatorname{grad}_{g} \rho\left(|\tau(\phi)|^{2}\right) .
\end{aligned}
$$

Since $M$ is compact, $|\tau(\phi)|^{2}$ attains its maximum at $p_{0}$. Evaluating the last equation at this point, shows $|\tau(\phi)|^{2}\left(p_{0}\right)=0$, therefore everywhere.

Furthermore, $0=\tau(\phi)=-2 d \phi\left(\operatorname{grad}_{g} \rho\right)$ and $\operatorname{rank} \phi=4$, imply that $\rho$ is constant.

Proposition 3.15. A map $\phi:\left(M^{4}, g\right) \rightarrow\left(N^{4}, h\right), M$ compact, with $S_{2}=0$ and $\operatorname{rank} \phi=4$, is harmonic. 
Proof. As $d \phi_{p}: T_{p} M \rightarrow T_{\phi(p)} N$ is an isomorphism at any point, there exists a unique vector field $Z$ such that $d \phi_{p}(Z)=\tau(\phi)(p), \forall p \in M$ and Equation (3.1), with $X=Y=Z$, reads

$$
\begin{aligned}
0 & =\frac{1}{2}|\tau(\phi)|^{2}|Z|^{2}+2\left\langle\nabla_{Z} \tau(\phi), \tau(\phi)\right\rangle \\
& =\frac{1}{2}|\tau(\phi)|^{2}|Z|^{2}+Z\left(|\tau(\phi)|^{2}\right) .
\end{aligned}
$$

Therefore the maximum of $|\tau(\phi)|^{2}$ must be zero.

By Proposition 3.4 if $\phi:\left(M^{4}, g\right) \rightarrow(N, h)$ has $\operatorname{rank} \phi \leq 3, S_{2}=0$ is equivalent to harmonicity.

\section{Maps With PARALlel STRESS-ENERGy TENSOR}

This section is dedicated to maps with parallel stress-energy tensor. Before we study in details the condition $\nabla S_{2}=0$, we show that, in certain circumstances, this condition is equivalent to $\operatorname{div} S_{2}=0$. Indeed, denoting by Riem $^{M}$ the sectional curvature of $M$, we have

Proposition 4.1. Let $\phi: M^{m} \rightarrow N^{m+1}$ be a Riemannian immersion with constant mean curvature. Assume that $N$ has constant sectional curvature, and $M$ is compact, orientable with $\operatorname{Riem}^{M} \geq 0$. Then $\operatorname{div} S_{2}=0$ if and only if $\nabla S_{2}=0$. Moreover, if $\operatorname{Riem}^{M}>0$, then $\operatorname{div} S_{2}=0$ if and only if $S_{2}=\lambda g, \lambda \in \mathbb{R}$, i.e. $M$ is umbilical.

Proof. Under the hypotheses on $\phi$ and $N$, the Codazzi equation becomes $\left(\nabla_{X} A_{H}\right)(Y)=\left(\nabla_{Y} A_{H}\right)(X), \forall X, Y \in C(T M)$. Thus, the tensor $S_{2}$, as an (1,1)-tensor on $M$, satisfies

$$
\left(\nabla_{X} S_{2}\right)(Y)=\left(\nabla_{Y} S_{2}\right)(X) .
$$

Now, applying a result of Berger (see, for example, [22, page 202]), the proposition follows.

If $\nabla S_{2}=0$ then clearly $\operatorname{div} S_{2}=0$ so, from Corollary [2.5] a submersion with parallel stress-energy tensor is biharmonic. Notice that Proposition 2.6 and $2.12 a$ ), give examples of submersions with $\nabla S_{2}=0$, and that Riemannian immersions with $\nabla S_{2}=0$ have normal bitension field.

Proposition 4.2. If a non-minimal Riemannian immersion $\phi:\left(M^{m}, g\right) \rightarrow$ $(N, h), m \neq 4$, has $\nabla S_{2}=0$, then $|\tau(\phi)|$ is constant.

Proof. For a Riemannian immersion:

$$
S_{2}(X, Y)=-\frac{1}{2}|\tau(\phi)|^{2}\langle X, Y\rangle+2\langle\tau(\phi), \nabla d \phi(X, Y)\rangle .
$$


Thus

$$
\begin{aligned}
0= & \left(\nabla S_{2}\right)(Z, X, Y)=\left(\nabla_{Z} S_{2}\right)(X, Y) \\
= & -\frac{1}{2} Z\left(|\tau(\phi)|^{2}\right)\langle X, Y\rangle+2(Z\langle\tau(\phi), \nabla d \phi(X, Y)\rangle \\
& \left.-\left\langle\tau(\phi), \nabla d \phi\left(\nabla_{Z} X, Y\right)\right\rangle-\left\langle\tau(\phi), \nabla d \phi\left(X, \nabla_{Z} Y\right)\right\rangle\right) .
\end{aligned}
$$

Take $p \in M$ and $\left\{X_{i}\right\}_{i=1}^{m}$ a geodesic frame around it, choose $X=Y=X_{i}$ and sum up, to obtain:

$$
\frac{4-m}{2} Z\left(|\tau(\phi)|^{2}\right)=0
$$

Remark 4.3. The hypothesis $m \neq 4$ is essential. Indeed, a pseudo-umbilical Riemannian immersion $\phi:\left(M^{4}, g\right) \rightarrow(N, h)$ satisfies $S_{2}=0$, hence $\nabla S_{2}=$ 0 , but $|\tau(\phi)|$ is not necessarily constant.

Theorem 4.4. A hypersurface $\mathbf{i}: M^{m} \rightarrow N^{m+1}, m \neq 4$, has $\nabla S_{2}=0$ if and only if it is parallel.

Proof. If $\nabla S_{2}=0,|\tau(\mathbf{i})|=m|H|$ is constant and $\tau(\mathbf{i})=c \eta$, where $c$ is constant and $\eta$ is a unit section of the normal bundle. Moreover,

$$
S_{2}(X, Y)=-\frac{c^{2}}{2}\langle X, Y\rangle+2 c b(X, Y),
$$

where $b(X, Y)=\langle\eta, \nabla d \mathbf{i}(X, Y)\rangle=\langle\eta, B(X, Y)\rangle$. We immediately infer that $\nabla S_{2}=0$ implies $\nabla b=0$, i.e. $M$ is parallel.

The converse is immediate, since $\nabla b=0$ implies $|H|$ constant.

Parallel hypersurfaces of space forms are classified ([13), and for the Euclidean sphere $\mathbb{S}^{m+1}$ parallel hypersurfaces are either hyperspheres $\mathbb{S}^{m}(a)$, $a \in(0,1]$, or Clifford tori $\mathbb{S}^{m_{1}}\left(a_{1}\right) \times \mathbb{S}^{m_{2}}\left(a_{2}\right)$, where $a_{1}^{2}+a_{2}^{2}=1, a_{1} \in(0,1)$, and $m_{1}+m_{2}=m$. Observe that hyperspheres are umbilical, while the Clifford tori are not.

Proposition 4.5. Let $\phi:(M, g) \rightarrow(N, h)$ be a non-minimal pseudoumbilical Riemannian immersion.

a) If $m=4$ then $S_{2}=0$, hence $\nabla S_{2}=0$.

b) If $m \neq 4, \nabla S_{2}=0$ if and only if $|\tau(\phi)|$ is constant.

Proof. Since $\phi$ is pseudo-umbilical, $\langle\nabla d \phi(X, Y), \tau(\phi)\rangle=\frac{1}{m}|\tau(\phi)|^{2}\langle X, Y\rangle$, consequently,

$$
S_{2}(X, Y)=\frac{4-m}{2 m}|\tau(\phi)|^{2}\langle X, Y\rangle
$$

The (pseudo-)umbilical hypersurfaces of space forms are classified [12, and have constant mean curvature. We indicate now a method to construct pseudo-umbilical submanifolds of constant mean curvature. 
Proposition 4.6. A minimal submanifold $\mathbf{j}: M^{m} \rightarrow P^{n-1}$ and a nonminimal umbilical hypersurface of constant mean curvature $\mathbf{i}: P^{n-1} \rightarrow N^{n}$ compose into a constant mean curvature pseudo-umbilical submanifold $\mathbf{i} \circ \mathbf{j}$ : $M \rightarrow N$.

Proof. Using the chain rule, the proof follows by a standard argument.

Remark 4.7. Take $N=\mathbb{S}^{n}, P=\mathbb{S}^{n-1}(a), a \in(0,1)$, and $M$ minimal in $\mathbb{S}^{n-1}(a)$, then the tangent part of $\tau_{2}(\mathbf{i} \circ \mathbf{j})$ vanishes. Moreover, $\mathbf{i} \circ \mathbf{j}$ is biharmonic if and only if $a=\frac{1}{\sqrt{2}}$ (10, 11]). Differently, replacing $N$ by $\mathbb{R}^{n}$ or $\mathbb{H}^{n}$ in the above construction, $\mathbf{i} \circ \mathbf{j}$ is never biharmonic $\left.(21]\right)$.

Remark 4.8. Let $\mathbf{i}: M \rightarrow N$ be a submanifold, the tangent part of $\tau_{2}(\mathbf{i})$ is (21]):

$$
\begin{aligned}
\left(\tau_{2}(\mathbf{i})\right)^{\top} & =-m\left(\frac{m}{2} \operatorname{grad}\left(|H|^{2}\right)+2 \operatorname{trace} A_{\nabla_{(\cdot)}^{\perp}}(\cdot)+2 \operatorname{trace}\left(R^{N}(d \mathbf{i} \cdot, H) d \mathbf{i} \cdot\right)^{\top}\right) \\
& =-m\left(-\frac{m}{2} \operatorname{grad}\left(|H|^{2}\right)+2 \operatorname{trace}\left(\nabla A_{H}\right)(\cdot, \cdot)\right) .
\end{aligned}
$$

A direct computation shows that, indeed, under the hypotheses of Theorem 4.4 and Proposition 4.5, $\tau_{2}(\mathbf{i})$ has vanishing tangent part.

\section{The CASE $S_{2}=\lambda g$}

The bienergy functional is homogeneous of degree zero with respect to the domain metric only in dimension four. Therefore, unless $m=4$, the bienergy can be made arbitrarily large or small by homotheties. To get around this problem, one considers variations with fixed volume. This type of variational problem is at the heart of Einstein metrics (see [6]).

Let $g$ be a Riemannian metric on a compact, orientable manifold $M$ and $\left\{g_{t}\right\}$ an isovolumetric variation of $g$, i.e. $\operatorname{Vol}\left(M, g_{t}\right)$ is constant, then

$$
0=\delta\left(\operatorname{Vol}\left(M, g_{t}\right)\right)=\frac{1}{2} \int_{M}\langle g, \omega\rangle v_{g}
$$

This says that $\omega$ is orthogonal to $g$ with respect to the $L^{2}$-scalar product on $T_{g} G=C\left(\odot^{2} T^{*} M\right)$. By Theorem [2.3, with respect to $\left\{g_{t}\right\}$ :

$$
\delta\left(F\left(g_{t}\right)\right)=-\frac{1}{2} \int_{M}\left\langle S_{2}, \omega\right\rangle v_{g}=-\frac{1}{2}\left(S_{2}, \omega\right) .
$$

Therefore, a critical point $g$ of $F$, with respect to isovolumetric variations, must be colinear with $S_{2}$, as vectors in $T_{g} G=C\left(\odot^{2} T^{*} M\right)$, i.e. $S_{2}=\lambda g$ for some $\lambda \in \mathbb{R}([\mathbf{7}, 24])$.

The trace of $S_{2}=\lambda g$ implies:

Proposition 5.1. Let $\phi:\left(M^{m}, g\right) \rightarrow(N, h)$ be a map with $S_{2}=\lambda g$. If one of the following holds
a) $m=2$,
b) $\operatorname{rank} \phi \leq m-1, m>2$,
c) $\phi$ is a submersion, $m>2$, 
then the norm of $\tau(\phi)$ is constant. Moreover, in case c) $\phi$ is biharmonic.

Proposition 2.6 and 2.12 a), give examples of submersions with $S_{2}=\lambda g$.

Proposition 5.2. If $\phi:\left(M^{m}, g\right) \rightarrow(N, h)$ satisfies $S_{2}=\lambda g$, with $M$ compact and orientable, then:

a) If $m=4, \lambda=0$.

b) If $m \neq 4, \frac{4-m}{2} \int_{M}|\tau(\phi)|^{2} v_{g}=\lambda m \operatorname{Vol}(M)$.

For Riemannian immersions we have the complete classification.

Theorem 5.3. For a non-minimal Riemannian immersion $\phi:\left(M^{m}, g\right) \rightarrow$ $(N, h), S_{2}=\lambda g$ if and only if $\phi$ is pseudo-umbilical and, if $m \neq 4$, the norm of $\tau(\phi)$ is constant.

Proof. Since the trace of $S_{2}=\lambda g$ gives:

$$
\frac{4-m}{2}|\tau(\phi)|^{2}=\lambda m
$$

if $m=4$, then $\lambda=0$ and $S_{2}$ vanishes, i.e. (Theorem 3.12) $\phi$ is pseudoumbilical.

If $m \neq 4$, (5.1) shows that $|\tau(\phi)|$ is constant. Then, replacing the value of $\lambda$ in the equation $S_{2}=\lambda g$, we obtain that $\phi$ is pseudo-umbilical.

Acknowledgements. The third author wishes to thank Professor Renzo Caddeo and the Dipartimento di Matematica e Informatica, Università di Cagliari, for hospitality during the preparation of this paper.

\section{REFERENCES}

[1] P. Baird, J. Eells. A conservation law for harmonic maps. Geometry Symposium, Utrecht 1980, 1-25, Lecture Notes in Math., 894, Springer 1981.

[2] P. Baird, D. Kamissoko. On constructing biharmonic maps and metrics. Ann. Global Anal. Geom. 23 (2003), 65-75.

[3] P. Baird, J.C. Wood. Harmonic Morphisms between Riemannian Manifolds. Oxford Science Publications, 2003.

[4] A. Balmuş. Biharmonic properties and conformal changes. An. Stiint. Univ. Al.I. Cuza Iasi Mat. (N.S.) 50 (2004), 361-372.

[5] A. Balmuş, S. Montaldo, C. Oniciuc. Biharmonic maps between warped product manifolds, preprint.

[6] M. Berger. Quelques formules de variation pour une structure riemannienne. Ann. Sci. Ec. Norm. Sup., 3 (1970), 285-294.

[7] A. Besse. Einstein Manifolds. Springer-Verlag, 1987.

[8] M. Bordoni. Spectral estimates for Schrödinger and Dirac-type operators on Riemannian manifolds. Math. Ann., 298 (1994), 693-718.

[9] Y.D. Burago, V.A. Zalgaller. Geometric Inequalities. Springer-Verlag, 1988.

[10] R. Caddeo, S. Montaldo, C. Oniciuc. Biharmonic submanifolds of $\mathbb{S}^{3}$. Int. J. Math., 12 (2001), 867-876.

[11] R. Caddeo, S. Montaldo, C. Oniciuc. Biharmonic submanifolds in spheres. Israel J. Math., 130 (2002), 109-123.

[12] B.Y. Chen. Geometry of Submanifolds. Marcel Dekker, 1973.

[13] F. Dillen. Semi-parallel hypersurfaces of a real space form. Israel J. Math., 75 (1991), 193-202. 
[14] J. Eells, J.H. Sampson. Harmonic mappings of Riemannian manifolds. Amer. J. Math., 86 (1964), 109-160.

[15] J. Eells, L. Lemaire. A report on harmonic maps. Bull. London Math. Soc., 10 (1978), $1-68$.

[16] D. Hilbert. Die grundlagen der physik. Math. Ann., 92 (1924), 1-32.

[17] G.Y. Jiang. 2-harmonic maps and their first and second variational formulas. Chinese Ann. Math. Ser. A, 7 (1986), 389-402.

[18] G.Y. Jiang. The conservation law for 2-harmonic maps between Riemannian manifolds. Acta Math. Sinica, 30 (1987), 220-225.

[19] The Bibliography of Biharmonic Maps.http://beltrami.sc.unica.it/biharmonic/

$[20]$ S. Montaldo, C. Oniciuc. A short survey on biharmonic maps between Riemannian manifolds. arXiv:math.DG/0510636

[21] C. Oniciuc. Biharmonic maps between Riemannian manifolds. An. Stiint. Univ. Al.I. Cuza Iasi Mat. (N.S.), 48 (2002), 237-248.

[22] P. Petersen. Riemannian Geometry. Springer-Verlag, 1998.

[23] T. Sakai. On Riemannian manifolds admitting a function whose gradient is of constant norm. Kodai Math. J., 19 (1996), 39-51.

[24] A. Sanini. Applicazioni tra varietà riemanniane con energia critica rispetto a deformazioni di metriche. Rend. Mat., 3 (1983), 53-63.

[25] S.T. Yau. Some function-theoretic properties of complete Riemannian manifold and their applications to geometry. Indiana Univ. Math. J., 25 (1976), 659-670.

Département de Mathématiques, Laboratoire CNRS UMR 6205, Université de Bretagne Occidentale, 6, avenue Victor Le Gorgeu, CS 93837, 29238 Brest Cedex 3, France

E-mail address: loubeau@univ-brest.fr

Università degli Studi di Cagliari, Dipartimento di Matematica e Informatica, Via Ospedale 72, 09124 Cagliari, Italia

E-mail address: montaldo@unica.it

Faculty of Mathematics, "Al.I. Cuza" University of Iasi, Bd. Carol I no. 11, 700506 IASI, ROMANIA

E-mail address: oniciucc@uaic.ro 